Research Paper

\title{
Prediction of Lymph Node Metastases in Gastric Cancer by Serum APE1 Expression
}

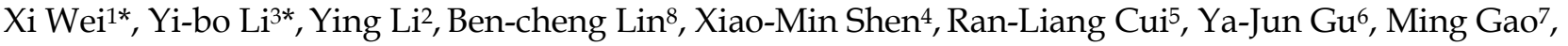 \\ Yue-Guo Li ${ }^{\square 凶}$, Sheng Zhang ${ }^{1 凶}$ \\ 1. Department of Diagnostic and Therapeutic Ultrasonography, Tianjin Medical University Cancer Institute and Hospital, National Clinical Research Center \\ for Cancer, Key laboratory of Cancer Prevention and Therapy, Tianjin's Clinical Research Center for Cancer, Tianjin, China; \\ 2. The Third Department of Breast Cancer, Tianjin Medical University Cancer Institute and Hospital, National Clinical Research Center for Cancer, Key \\ laboratory of Cancer Prevention and Therapy, Tianjin's Clinical Research Center for Cancer, Tianjin, China; \\ 3. Department of general surgery, The first affiliated hospital of DaLian medical university; \\ 4. Department of Clinical Laboratory, Tianjin second people' hospital; \\ 5. Department of Clinical Laboratory, Tianjin Medical University Cancer Institute and Hospital, National Clinical Research Center for Cancer, Key laboratory \\ of Cancer Prevention and Therapy, Tianjin's Clinical Research Center for Cancer, Tianjin, China \\ 6. School of Medical Laboratory, Tianjin Medical University; \\ 7. Department of Thyroid and Cervical Tumor, Tianjin Medical University Cancer Institute and Hospital, National Clinical Research Center for Cancer, Key \\ laboratory of Cancer Prevention and Therapy, Tianjin's Clinical Research Center for Cancer, Tianjin, China; \\ 8. Tianjin Institute of Health and Environmental Medicine, Key Laboratory of Risk Assessment and Control Technology for Environment \& Food Safety, \\ Tianjin 300050, China. \\ ${ }^{*} \mathrm{Xi}$ Wei and Yi-bo Li contributed equally to this work.
}

$\triangle$ Corresponding authors: Professor Sheng Zhang and Yue-Guo Li, MD. PhD.; Email: zs19620112@126.com; kesaqi@163.com. Telephone: +86-022-23340123. Department of Diagnostic and Therapeutic Ultrasonography, and Department of Clinical Laboratory, Tianjin Medical University Cancer Institute and Hospital, Tianjin, China. Huanhuxi Road, Hexi District, Tianjin, China 300060

(c) Ivyspring International Publisher. This is an open access article distributed under the terms of the Creative Commons Attribution (CC BY-NC) license (https://creativecommons.org/licenses/by-nc/4.0/). See http://ivyspring.com/terms for full terms and conditions.

Received: 2016.12.04; Accepted: 2017.03.06; Published: 2017.06.01

\begin{abstract}
Aims: To investigate the functional role of serum Human apurinic/apyrimidinic endonuclease 1 (APEI) in prediction of lymph node metastasis in gastric cancer patients.

Materials and methods: Serum samples were pre-operational collected from 86 patients with gastric cancer from Tianjin Medical University Cancer Institute and Hospital from March 2016 to August 2016. The serum of APEI was measured by ELISA development kit and other CA242, CA724, CA199 and CEA levels by electrochemiluminescence assay.

Results: The total of 86 patients with gastric cancer was classified into two groups (lymph node positive and negative groups). Using ELISA assay, we found out that the concentration of serum APEl was higher in lymph node positive group than that of lymph node negative group. The receiver operating characteristic (ROC) curve was performed to analyze, indicating that area under the ROC curve of serum APEl were better than those of each regular markers (CEA+CA199+CA242+CA724) or combination of these markers. Additionally, the APE1 overexpression was uncovered in tissue of gastric cancer patients with lymph nodes metastases, which is correlation with results of serum APE1.
\end{abstract}

Conclusion: Serum APEI was identified as a valuable marker for prediction of lymph node metastases in patients with gastric cancer.

Key words: APE1, Gastric cancer, lymph node involvement, serum, marker.

\section{Introduction}

Apurinic/apyrimidinic endonuclease (APE1) is a multifunctional protein, which is not only involved in DNA damage repair but regulates redox activity related to kinds of tumor occurrence and development [1]. Our previous study indicated that APE1 overexpression was associated with poor 
prognosis of non-small cell lung cancer and enhanced epithelial-Mesenchymal Transition (EMT) of lung cancer cell in vitro and in vivo [2]. Other study also reported that APE1 inhibitor was able to suppress gastric cancer cell migration and invasion in combination with 5-Fu treatment [3]. Moreover, APE1 serum protein has been screened as candidate biomarkers for lung cancer in prediction of chemotherapeutic efficacy [4].

Gastric cancer is one of most high mortality carcinoma in patients with poor prognosis [5]. Screening early and efficacy biomarkers is critical for physicians to predict gastric cancer patients' metastasis and prognosis [6]. Potential new serum maker candidates include vascular endothelial growth factor, cytokines and growth factors and other independent clinical prognostic predictors [7]. Serum autoantibodies used as biomarkers are highly stable in blood, such as serum Her-2, serum Helicobacter pylori KatA and AhpC antibodies, for novel biomarkers in gastric cancer [8]. Our study found that APE1 protein overexpression is associated with poor overall survival of gastric cancer patients [3]. In this study, based on our previous results, we aim to explore the relationship between serum APE1 expression and gastric cancer lymph nodes involvement [3]. We investigate the role of serum APE1 in further prediction of gastric cancer metastasis and prognosis.

DNA damage repair plays important roles in maintaining genomic stability but increases the progression in cancer development. APE1 protein is seen as one of the essential DNA repair proteins involving in cell survival and other carcinogenesis [1]. Abnormally abundant APE1 has been identified expression in serum in NSCLC in previous study [9]. To our knowledge, the function of serum APE1 in prediction of prognosis in gastric cancer is less clear. In our study, using ELISA assay, we speculate that serum APE1 might be correlation with diagnostic efficacy and lymph node metastases in gastric cancer patients.

\section{Materials and Methods}

The study was approved by the Research Ethics Committee of Tianjin Medical University Cancer Institute and Hospital. Informed consent was obtained from all of the patients. All specimens were handled and made anonymous according to ethical and legal standards. Serum samples were collected before operation from 86 patients with gastric cancer in Tianjin Medical University Cancer Institute and Hospital from March 2016 to August 2016. All patients with clear gastric cancer pathology did not receive neoadjuvant chemotherapy, radiation therapy, or immunotherapy before surgery. Table 1 summarizes the clinicopathological data of these patients with gastric cancer.

Table 1. The characteristics of patients with gastric cancer for serum APEl examination

\begin{tabular}{llll}
\hline $\begin{array}{l}\text { Patients } \\
\text { characteristics }\end{array}$ & NO. & LN negative & LN positive \\
\hline Total & 86 & 35 & 52 \\
Gender & male & 25 & 39 \\
& female & 10 & 13 \\
Age (year) & & 57.9 & 58.7 \\
Stage & I-II & $31(88.5 \%)$ & $5(9.6 \%)$ \\
& III-IV & $4(11.5 \%)$ & $47(90.4 \%)$ \\
Gastric location & cardia & $5(1.4 \%)$ & $12(2.3 \%)$ \\
& body & $7(2.0 \%)$ & $10(1.9 \%)$ \\
& antrum & $15(42.9 \%)$ & $24(4.6 \%)$ \\
& angulus & $7(2.0 \%)$ & $2(3.8 \%)$ \\
TNM & pylorus & $1(0.3 \%)$ & $4(7.7 \%)$ \\
& T1-2/T3-4 & $22 / 13(62.9 \% / 37.1 \%)$ & $5 / 47(9.6 \% / 90.4 \%)$ \\
& N0-1/N2-3 & $0 / 0(0 \% / 0 \%)$ & $22 / 30(42.3 \% / 57.7 \%)$ \\
Tumor size & M0/M1 & $0 / 0(0 \% / 0 \%)$ & $48 / 4(92.3 \% / 7.7 \%)$ \\
& Average size & 3.52 & 5.35 \\
Lymph node & (cm) & & \\
metastases & Average NO. & 0 & 15 \\
Her-2 status & positive & $3(8.6 \%)$ & $17(32.7 \%)$ \\
\hline LN: lymph node; NO. number & &
\end{tabular}

CA242, CA724, CA199 and CEA levels were obtained from medical records during the inpatient periods. Measurement of Human apurinic/ apyrimidinic endonuclease 1 (APE1) using ELISA development kit (abbexa company, Cambridge, UK) was used to measure APE1 levels in human serum; 96-well ELISA microplates were coated overnight with $100 \mathrm{Ml}$ of APE1 antibody at a final concentration of $0.25 \mathrm{mg} / \mathrm{L}$ in phosphate-buffered saline (PBS). After washing with $0.05 \%(\mathrm{w} / \mathrm{v})$ Tween-20 in PBS (PBST, pH 7.4), the wells were blocked with blocking buffer at room temperature for $1 \mathrm{~h}$. Then, $100 \mu \mathrm{L}$ of diluted serum samples (1:5 dilutions) were added and incubated at room temperature for $2 \mathrm{~h}$. Similarly, $100 \mu \mathrm{L}$ of PBST lacking antibody was used as a negative control. Following three washes with PBST, $100 \mu \mathrm{L}$ of antibody diluted to a concentration of 0.25 $\mathrm{mg} / \mathrm{L}$ was added. After incubation at room temperature for $2 \mathrm{~h}, 100 \mu \mathrm{L}$ of avidin-horseradish peroxidase-conjugated secondary antibody (1:2000 dilutions) was added, and plates were incubated at room temperature for $30 \mathrm{~min}$. The excess conjugate was removed by washing the plates three times with PBST. The amount of bound conjugate was determined by adding ABTS liquid substrate solution to each well, and plates were incubated at room temperature for color development. The absorbance was measured at $405 \mathrm{~nm}$ using a Model 680 microplate reader (Bio-Rad Lab. Inc., Hercules, CA, 
USA). All analyses were performed in triplicate. The coefficient of variation was lower than $15 \%$ between analyses.

In the IHC staining assay, the protocol of previous study was followed (3). The primary antibody against APE1 (Cell signaling, MA) was used for samples staining.

Statistical significance was determined with the nonparametric Mann-Whitney U test (for differences between two groups) with SPSS 16.0 software. The receiver operating characteristic (ROC) curve was made using Medcalc software. Values of $p<0.05$ were considered statistically significant.

\section{Results}

\section{Characteristics of patients with gastric carcinoma}

Table 1 showed that the characteristics of 86 patients with gastric carcinoma in Tianjin cancer hospital. The stage, TNM and tumor location, Her-2 status in lymph node positive and negative groups were all analyzed.

\section{Serum APE1 predicts lymph node metastases in patients with gastric cancer}

To investigate the role of serum APE1 in prediction of gastric cancer with lymph node metastases, we tested the level of serum APE1 in two different groups (lymph node positive vs negative group). As data shown in plot, we found out that the concentration of serum APE1 in lymph node metastases group was higher than that of lymph node negative group $(676.7 \pm 641.5$ vs $386.7 \pm 360.5 \mathrm{ng} / \mathrm{L})$ $(P=0.023)$ (Figure 1). This finding indicated that serum APE1 maker is able to predict the lymph node involvement in patients with gastric cancer.

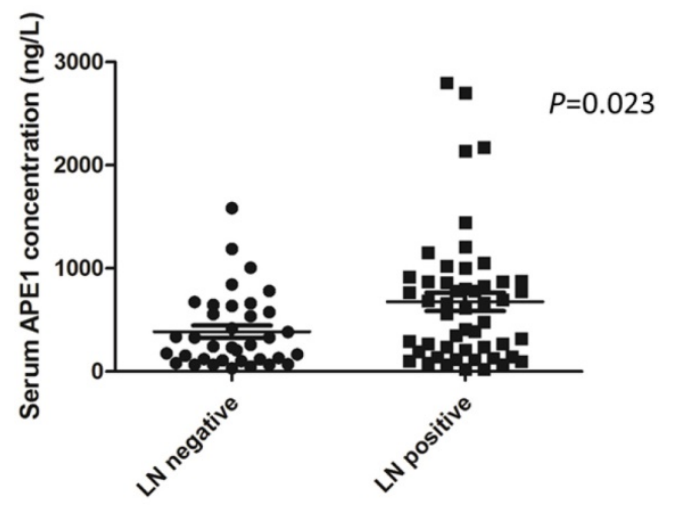

Figure 1. Serum APE1 level in gastric cancer patients in lymph node positive and negative groups assessed by ELISA. Among 86 gastric cancer patients, the mean $\pm S D$ of concentration of serum APEl was $676.7 \pm 641.5$ in lymph node metastases group and $386.7 \pm 360.5 \mathrm{ng} / \mathrm{L}$ in lymph node negative group $(P=0.023$, t-test).
The diagnostic performance of serum APEI compared with other serum makers in gastric cancer

Although serum APE1 was found out to be effective maker for prediction of lymph node metastases, other serum makers (CEA, CA199, CA242 and CA724) has been presented as regular makers for gastric cancer diagnosis. In our study, we compared serum APE1 and other makers to investigate the diagnostic performance of serum APE1 in diagnosis of gastric lymph node metastases. The receiver operating characteristic (ROC) curve of serum APE1 were better than those of other markers (Figure 2). As Table 2 shown, the specificity of APE1 (85.7\%) was higher than CA242, CA724 and CEA (37.1\%, 57.1\% and $82.9 \%)$, but lower than that of CA199 (97.1\%) in prediction of lymph node metastases. The cutoff value of APE1 was analyzed as 660.2. However, we found that serum APE1 has better diagnostic performance than that of each markers and even combination of other four markers (CEA+CA199+CA242+CA724) (Figure 3). These data indicated that serum APE1 not only predicates lymph node metastases in gastric cancer but contributes to good performance in diagnosis.

Table 2. The diagnostic value of APE1, CA199, CA242, CA724 and CEA in prediction of gastric cancer with lymph node metastasis

\begin{tabular}{llllll}
\hline & APE1 & CA199 & CA242 & CA724 & CEA \\
\hline Sensitivity & $49 \%$ & $19.6 \%$ & $84.3 \%$ & $58.8 \%$ & $51.0 \%$ \\
Specificity & $85.7 \%$ & $97.1 \%$ & $37.1 \%$ & $57.1 \%$ & $82.9 \%$ \\
Cutoff value & 660.2 & 33.17 & 2.68 & 2.02 & 3.18 \\
\hline
\end{tabular}

\section{High expression of APE 1 in tissue and serum of patients with gastric cancer}

We also search out the dataset of The Cancer Genome Atlas (TCGA) to have results of APE1 overexpression in tissues of primary site and lymph node metastases in 129 patients with gastric cancer (Figure 5). Among our patients, APE1 expression in tissue samples was stained in 65 samples of gastric cancer, comparing with control tissues. In this study, we find out APE1 has higher expression in gastric cancer with lymph node metastases than that of negative lymph node group $\left(\chi^{2}=5.128, P=0.044\right)$ (Table $3)$, with a significant correlation with results of serum APE1 expression within the same cohort $(\mathrm{r}=0.262$ $P=0.035$ ) (Table 4 and Figure 4 ). 

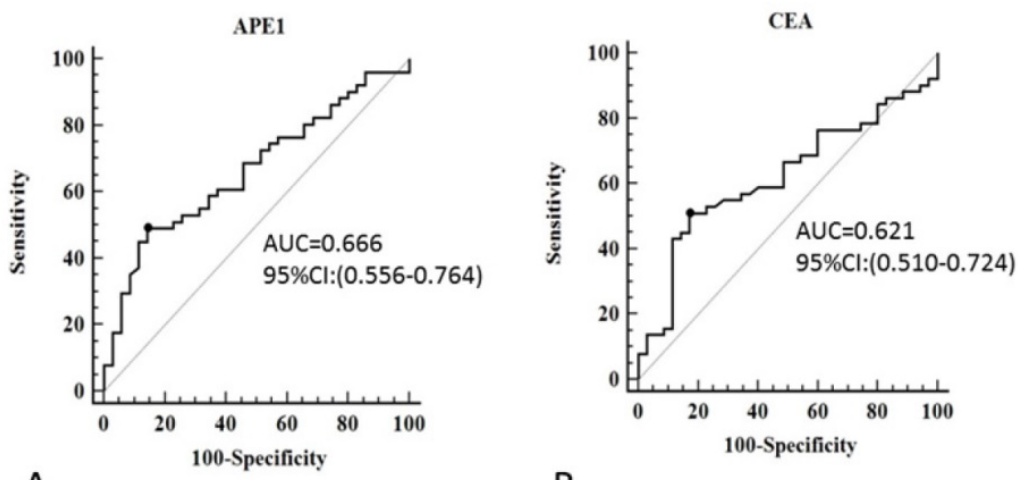

A

B
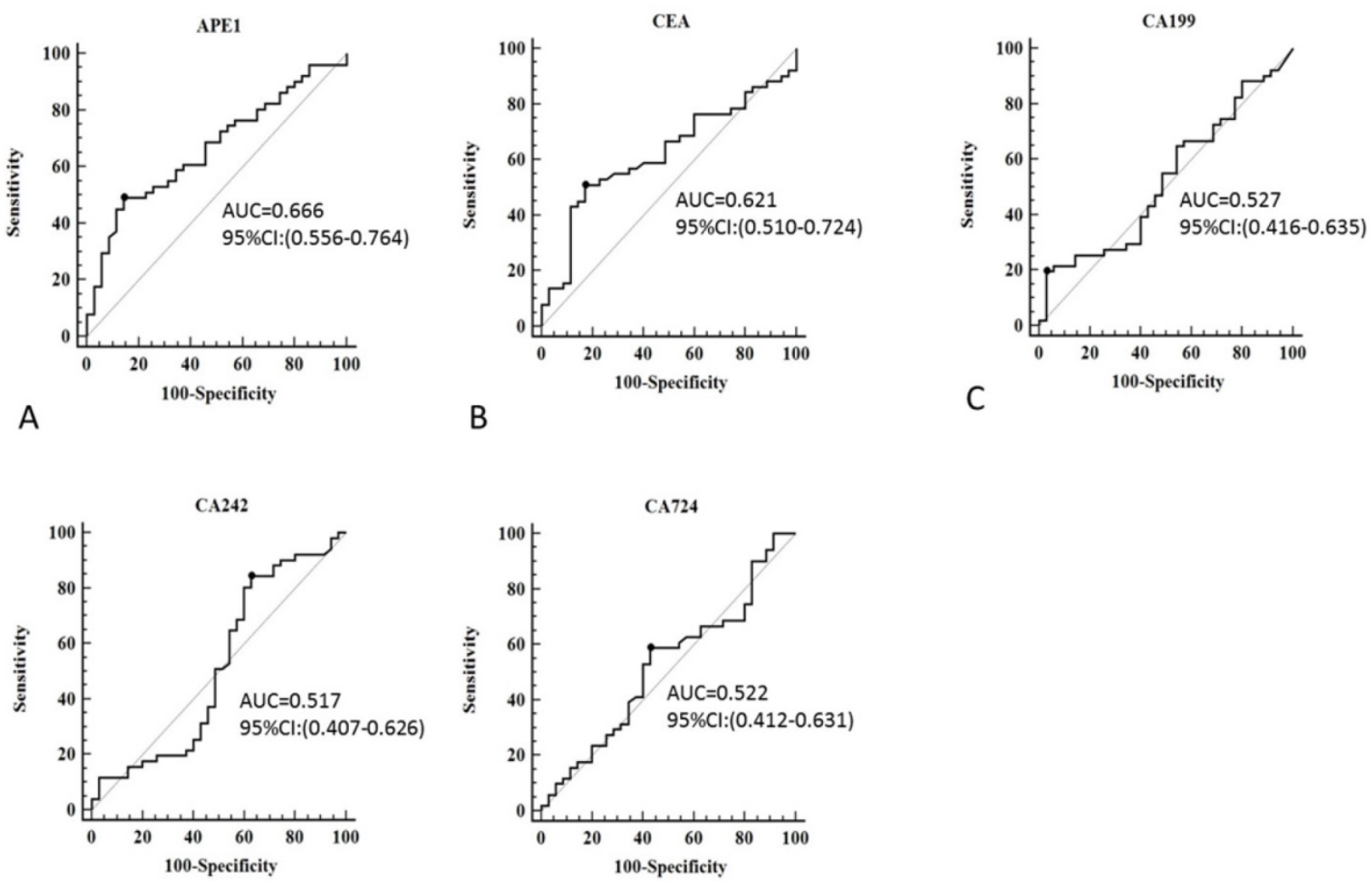

D

$E$

Figure 2. Receiver operating characteristic curve of serum APE1, CEA, CA199, CA242 and CA724 for diagnostic analysis in all subjects. A: APE1, B: CA199, C: CA242 and D: CA724.

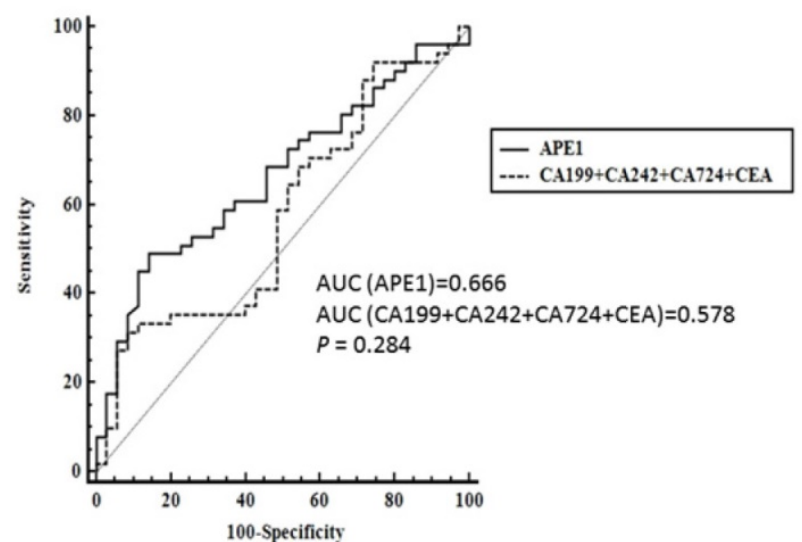

A

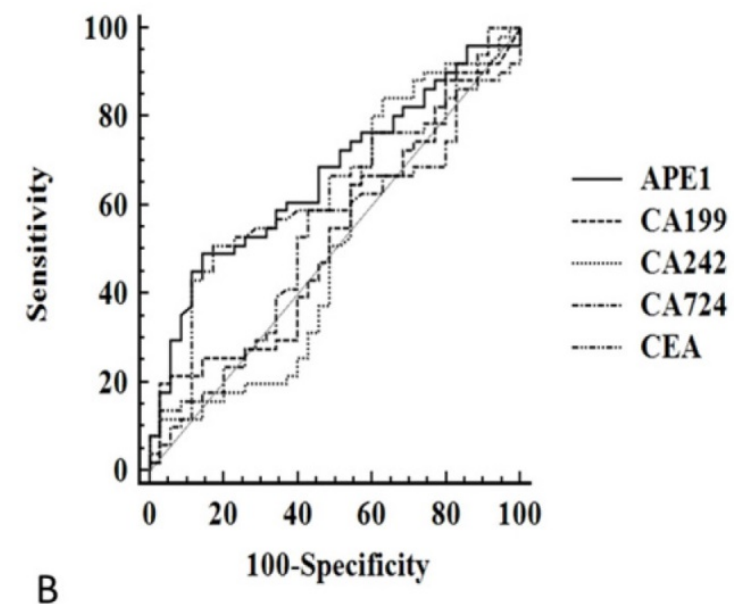

Figure 3. Receiver operating characteristic curve of serum APE1 and combination of CEA, CA199, CA242 and CA724 for diagnostic analysis in all subjects. A: APE1/CEA/CA199/CA242/CA724; B. APE1/ CEA+CA199+CA242+CA724.

Table 3. The APE1 tissue expression in prediction of gastric cancer patients with lymph node metastases

\begin{tabular}{|c|c|c|c|c|c|c|}
\hline \multirow[t]{2}{*}{ Samples } & & \multicolumn{2}{|c|}{ APE1 expression } & \multirow[t]{2}{*}{ total } & \multirow[t]{2}{*}{$\chi^{2}$} & \multirow[t]{2}{*}{$P$} \\
\hline & & positive & negative & & & \\
\hline \multirow[t]{3}{*}{$\overline{\mathrm{LN}}$} & positive & 47 & 3 & 50 & 5.128 & 0.044 \\
\hline & negative & 11 & 4 & 15 & & \\
\hline & Total & 58 & 7 & 65 & & \\
\hline
\end{tabular}

LN: lymph node; NO. number
Table 4. The correlation of APEI tissue expression and serum results in gastric cancer patients

\begin{tabular}{cllllll}
\hline Samples & \multicolumn{2}{l}{ APE1 expression } & total & r value & $P$ \\
\cline { 2 - 4 } & positive & negative & & & \\
\hline APE1 serum positive & 40 & 2 & 42 & 0.262 & 0.035 \\
negative & 18 & 5 & 23 & & \\
Total & 58 & 7 & 65 & & \\
\hline
\end{tabular}

LN: lymph node; NO. number 
HE

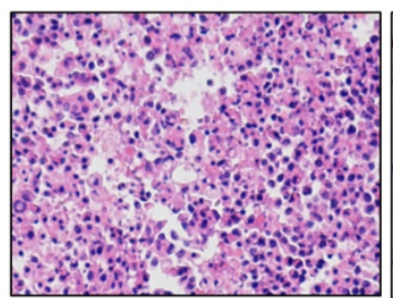

A

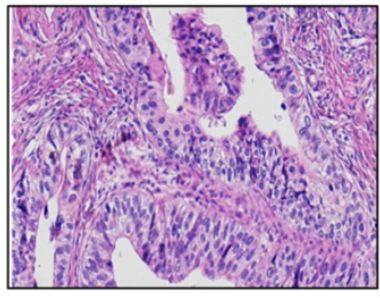

C

Figure 4. Immunohistochemistry (IHC) analyses of APE1 expression in gastric cancer tissues (A. and D. Hematoxylin-eosin (HE) staining; B. Poor-differentiated carcinoma; C. adenocarcinoma, magnification 200X);

\section{Discussion}

Gastric carcinoma develops from the lining of stomach and spreads to other parts of the body, such as lymph nodes, liver, lungs and bone [10]. The diagnosis of gastric cancer was done by biopsy with endoscopy. The screening effort will reduce poor prognosis of advanced gastric cancer. The four serum cancer markers, such as CEA, CA199, AFP and CA125, has been examined as the prognostic value in gastric cancer patients [11]. In our study, we investigated the role of serum APE1 in prediction of lymph node metastases in gastric cancer.

APE1 has two different functions, DNA repair and redox activity, which is beneficial to cellular process and biological development [12]. A researcher from Japan first reported APE1 autoantibodies in serum from systemic lupus erythematosus patients [13]. APE1 has been identified to be an ectopic protein from tumor tissue into serum. Wang et al. reported that APE1 autoantibodies by indirect ELISA are monitored in NSCLC patients' blood to be a potential tumor marker [9]. Another study showed APE1/Ref-1 to be a potential bladder cancer biomarker in patient's urine for clinical diagnosis of bladder cancer [14]. One research group uncovered extracellularly secreted APE1/Ref-1 triggers apoptosis in triple-negative breast cancer, indicating its secretion into the circulation probably through secreted formation from intracellular into extra circulation. In our study, we tested serum APE1 in patients with gastric cancer before operation. After grouping into lymph node positive and negative cohorts, we found out that the average value of serum APE1 is higher in gastric cancer with lymph node metastases than control group. To investigate the diagnostic performance of serum APE1 using ELISA detection, the serum CEA, CA199, CA242 and CA724 were compared. The data from ROC curve showed that the diagnostic value of serum APE1 was better than each regular serum markers, and even than combination of these four markers. In this case, we concluded that serum APE1 has good diagnostic performance in patients with gastric cancer with lymph node metastases.

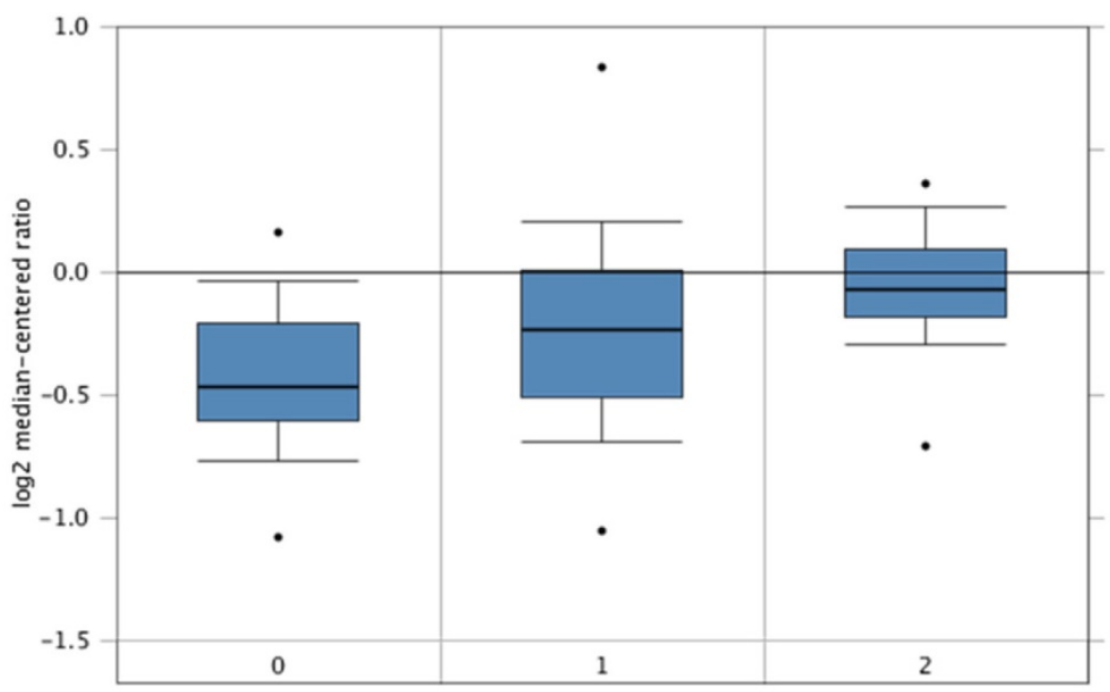

0. No value (29)

1. Primary Site (86) 2. Lymph Node Metastasis (14)

Figure 5. The APE1 expression in 129 patients with gastric cancer in primary site (86) and lymph node metastasis (14) and No value (29) analyzed from TCGA dataset. 
Using immunohistorchemistry (IHC) to detect APE1 expression, multiple studies reported that APE1 positive expression associated with poor prognosis in various cancers. Some studies also evaluated the predictors of gastric cancer risk with APE1 ploymorphism $[15,16]$. Lymph node involvement has been considered as independent factors for advanced gastric cancers [6]. Prediction of lymph node metastases in gastric cancer plays a critical role in preoperative strategy management [10]. Peripheral blood examination of APE1 enable to assist lymph node metastases assessment other than imaging evaluation. Our previous study indicated that APE1 inhibitor has synergetic effect to promote 5-FU based therapy in gastric cancer [3]. We also search out the TCGA dateset to confirm our findings. The results demonstrated that APE1 overexpression was shown in gastric cancer tissue and metastatic lymph nodes, which is consistent with our studies on gastric cancer tissue in IHC staining.

In conclusion, we identified serum APE1 being a good marker for prediction of lymph node metastases in patients with gastric cancer. The sensitivity and specificity of serum APE1 are both higher than those of regular serum markers. A statistical significant correlation was found between APE1 expression in tissue and peripheral blood and lymph node involvement in advanced gastric cancer. These findings provide clues that serum APE1 possess a potential to be a serum diagnostic marker for advanced gastric cancer. Further work should focus on the prediction value on serum APE1 in prognosis of gastric cancer in a large cohort.

\section{Acknowledgments}

This work was supported by research grants from National Scientific Foundation of China (\#81401412), Tianjin Research Program of Application Foundation and Advanced Technology (\#15JCQNJC11600), Tianjin City High School Science \& Technology Fund Planning Project (\#20140124)

\section{Competing Interests}

The authors have declared that no competing interest exists.

\section{References}

1. Abbotts $\mathrm{R}$ and Madhusudan S. Human AP endonuclease 1 (APE1): from mechanistic insights to druggable target in cancer. Cancer treatment reviews. 2010; 36(5):425-435.

2. Wei X, Li O, Li Y, Duan W, Huang C, Zheng X, Sun L, Luo J, Wang D, Zhang S, Xin $X$ and Gao M. Prediction of survival prognosis of non-small cell lung cancer by APE1 through regulation of Epithelial-Mesenchymal Transition. Oncotarget. 2016; 7(19):28523-28539.

3. Wei X, Duan W, Li Y, Zhang S, Xin X, Sun L, Gao M, Li Q and Wang D. AT101 exerts a synergetic efficacy in gastric cancer patients with 5-FU based treatment through promoting apoptosis and autophagy. Oncotarget. 2016.

4. Wang D, Xiang DB, Yang XQ, Chen LS, Li MX, Zhong ZY and Zhang YS. APE1 overexpression is associated with cisplatin resistance in non-small cell lung cancer and targeted inhibition of APE1 enhances the activity of cisplatin in A549 cells. Lung cancer (Amsterdam, Netherlands). 2009; 66(3):298-304.

5. Torre LA, Bray F, Siegel RL, Ferlay J, Lortet-Tieulent J and Jemal A. Global cancer statistics, 2012. CA: a cancer journal for clinicians. 2015; 65(2):87-108.

6. Jiang Y, Kimchi ET, Montero AJ, Staveley-O'Carroll KF and Ajani JA. Upper gastrointestinal tumors: current status and future perspectives. Expert review of anticancer therapy. 2008; 8(6):975-991.

7. Bharti A, Ma PC and Salgia R. Biomarker discovery in lung cancer--promises and challenges of clinical proteomics. Mass spectrometry reviews. 2007; 26(3):451-466.

8. Zhang B, Li HL, Fan Q, Guo F, Ren XY, Zhou HB, Zhu JW, Zhao YS and Tian WJ. Serum Helicobacter pylori KatA and AhpC antibodies as novel biomarkers for gastric cancer. World journal of gastroenterology. 2016; 22(21):5060-5067.

9. Dai N, Cao XJ, Li MX, Qing Y, Liao L, Lu XF, Zhang SH, Li Z, Yang YX and Wang D. Serum APE1 autoantibodies: a novel potential tumor marker and predictor of chemotherapeutic efficacy in non-small cell lung cancer. PloS one. 2013; 8(3):e58001.

10. Orditura M, Galizia G, Sforza V, Gambardella V, Fabozzi A, Laterza MM, Andreozzi F, Ventriglia J, Savastano B, Mabilia A, Lieto E, Ciardiello F and De Vita F. Treatment of gastric cancer. World journal of gastroenterology. 2014; 20(7):1635-1649.

11. Feng F, Sun L, Liu Z, Liu S, Zheng G, Xu G, Guo M, Lian X, Fan D and Zhang $\mathrm{H}$. Prognostic values of normal preoperative serum cancer markers for gastric cancer. Oncotarget. 2016.

12. Demple B, Herman $\mathrm{T}$ and Chen DS. Cloning and expression of APE, the cDNA encoding the major human apurinic endonuclease: definition of a family of DNA repair enzymes. Proceedings of the National Academy of Sciences of the United States of America. 1991; 88(24):11450-11454.

13. Katsumata Y, Kawaguchi Y, Baba S, Hattori S, Tahara K, Ito K, Iwasaki T, Yamaguchi N, Oyama M, Kozuka-Hata H, Hattori H, Nagata K, Yamanaka H and Hara M. Identification of three new autoantibodies associated with systemic lupus erythematosus using two proteomic approaches. Molecular \& cellular proteomics : MCP. 2011; 10(6):M110.005330.

14. Choi S, Shin JH, Lee YR, Joo HK, Song KH, Na YG, Chang SJ, Lim JS and Jeon BH. Urinary APE1/Ref-1: A Potential Bladder Cancer Biomarker. Disease markers. 2016; 2016:7276502.

15. Gu D, Wang M, Wang S, Zhang Z and Chen J. The DNA repair gene APE1 T1349G polymorphism and risk of gastric cancer in a Chinese population. PloS one. 2011; 6(12):e28971.

16. Al-Attar A, Gossage L, Fareed KR, Shehata M, Mohammed M, Zaitoun AM, Soomro I, Lobo DN, Abbotts R, Chan S and Madhusudan S. Human apurinic/apyrimidinic endonuclease (APE1) is a prognostic factor in ovarian, gastro-oesophageal and pancreatico-biliary cancers. British journal of cancer. 2010; 102(4):704-709. 